\title{
Effect of raised NEFA levels on endothelial function is modified by fatty acid composition
}

\author{
A. K. Thompson, K. J. Newens, K. G. Jackson and C. M. Williams \\ Department of Food Biosciences, University of Reading, Reading RG6 6AP, UK
}

Increasing NEFA has been shown to impair endothelial function ${ }^{(1)}$, but this has not been previously investigated in a controlled study using NEFA enriched in different types of fatty acid. Endothelial dysfunction is associated with the CVD ${ }^{(2)}$, and a number of techniques have been developed to measure this. Flow-mediated dilatation (FMD) is considered the most reliable of the non-invasive options ${ }^{(3)}$, but other commonly used techniques include laser Doppler iontophoresis (LDI) and digital volume pulse (DVP). All these measure slightly different aspects of endothelial function in different vascular beds.

Fifty-six healthy subjects were given drinks either rich in SFA $(0.42 \mathrm{~g}$ palm stearin per kg bodyweight) or SFA and $n-3$ PUFA (0.36 g palm stearin $+0.06 \mathrm{~g}$ DHA-rich fish oil per kg bodyweight) on separate occasions. The drinks were given as a bolus $(\approx 33 \%$ of total fat) followed by smaller amounts $(\approx 11 \%)$ every $30 \mathrm{~min}$ for $240 \mathrm{~min}$. After $60 \mathrm{~min}$, an intravenous infusion of heparin was used to increase NEFA levels. Endothelial function was measured at baseline and $240 \mathrm{~min}$. Blood samples were taken every 30 min to monitor changes in NEFA.

The increase in total plasma NEFA levels as a result of consumption of the chocolate drinks and the heparin infusion were the same on both study days (Fig. 1). Following the consumption of SFA, there was a reduction in the FMD response (impaired endothelial function), while there was an increase in FMD (enhanced endothelial function) with SFA and $n$-3 PUFA (Fig. 2). There were no significant differences in LDI or DVP between study days, suggesting that there was no effect of NEFA type on the microvasculature (as measured by LDI) or on aspects of arterial stiffness (DVP).

Our findings suggest that the effect of raised NEFA on endothelial function may be dependent on the fatty acid composition of NEFA rather than simply the total NEFA concentration, and moderate substitution of $n-3$ PUFA for SFA may have a positive effect on endothelial function at high NEFA levels.
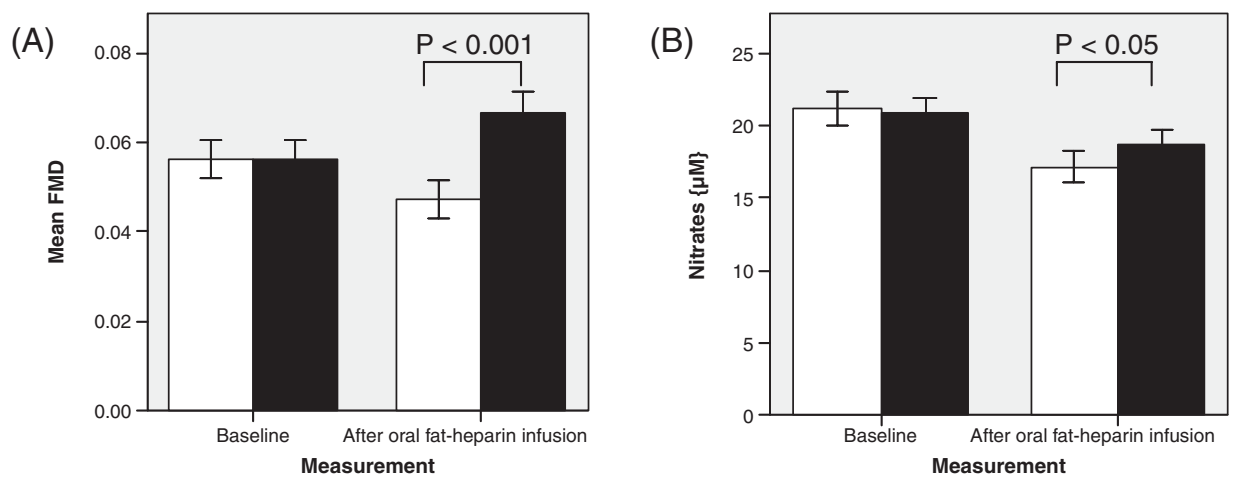

Fig. 1. The FMD response (A, $n$ 56) and nitric oxide level (B, $n$ 36) at baseline and after $3.5 \mathrm{~h}$ oral feeding of chocolate drink containing particular fat (SFA = white bars; $\mathrm{SFA}+n 3 \mathrm{PUFA}=$ black bars) and $2.5 \mathrm{~h}$ of intravenous heparin infusion (mean (SE)).

The authors gratefully acknowledge funding from BBSRC, Unilever and FRST (New Zealand).

1. Kim F et al. (2005) Arterioscler Thromb Vasc Biol 25, 989-994.

2. Widlansky ME et al. (2003) J Am Coll Cardiol 42, 1149-1160.

3. Moens AL et al. (2005) Chest 127, 2254-2263. 